\title{
The helium-weak silicon star HR 7224
}

\section{Radial velocity and line profile variations ${ }^{\star}, \star \star$}

\author{
H. Lehmann ${ }^{1}$, V. Tsymbal ${ }^{2}$, D. E. Mkrtichian ${ }^{3,4}$, and L. Fraga ${ }^{1}$ \\ 1 Thüringer Landessternwarte Tautenburg, 7778 Tautenburg, Germany \\ e-mail: lehm@tls-tautenburg.de \\ 2 Tavrian National University Dep. Astronomy, Simferopol, Ukraine; Institut für Astronomie, Universität Wien, \\ Türkenschanzstraße 17, 1180 Wien, Austria \\ e-mail: vad@simfi.net \\ 3 Astrophysical Research Center for the Structure and Evolution of the Cosmos, Sejong University, Seoul 143-747, Korea \\ 4 Astronomical Observatory, Odessa National University, Shevchenko Park, 65014, Odessa, Ukraine \\ e-mail: david@arcsec.sejong.ac.kr
}

Received 26 April 2006 / Accepted 22 June 2006

\section{ABSTRACT}

\begin{abstract}
Aims. HR 7224 is a rapidly rotating He-weak silicon star known as photometrically variable with a period of $1.123 \mathrm{~d}$. Drastic changes of photometric amplitude and period were reported in 2003. We investigate the star spectroscopically to determine the time scales of variability, to derive basic atmospheric parameters, and to give first estimates on possible surface structures.

Methods. We investigate time series of more then 570 high-resolution spectra of HR 7224 for radial velocity and line profile variations. The rotation period is determined from period analysis of data. Comparing radial velocity and line profile variations we draw first conclusions on possible surface structures in terms of line strengths. From Balmer and metal lines we determine $T_{\text {eff }}, \log g, v \sin i$, and metal abundances depending on rotation phase.

Results. The star shows a remarkable amplitude of radial velocity variation of $\pm 6 \mathrm{~km} \mathrm{~s}^{-1}$. From this variation we deduce a rotation period of $1.12324 \mathrm{~d}$. The period and the double-wave shaped radial velocity curve are in agreement with earlier photometric findings. The observed radial velocity variations can be explained by the rotation period and its harmonics and no further periodic contributions were found in the residuals. HR 7224 has enhanced $\mathrm{Si}$ and Fe abundances whereas He is extremely depleted. The star shows line strength variations of all observed elements with the rotation period. Variations of radial velocity, line strength and line shape of silicon lines and their relationships can be explained by assuming two large spots on the stellar surface showing enhanced line strength. From Balmer lines we deduce different temperatures of the star for different rotation phases. The observed difference in amplitudes of the variation of $\mathrm{Mg}$ II and $\mathrm{Si}$ II lines supports the assumption of surface regions of different temperature on this presumably magnetic $\mathrm{Cp}$ star. Synthetic uvby colors computed from adapted synthetic spectra confirm the high photometric amplitude reported for the 2003 light curves.
\end{abstract}

Key words. stars: chemically peculiar - stars: variables: general - stars: atmospheres - stars: abundances stars: individual: HR 7224

\section{Introduction}

HR 7224 (HD 177410, HIP 93187, EE Dra) is a rapidly rotating $\mathrm{Cp} \mathrm{Si} \mathrm{star.} \mathrm{It} \mathrm{was} \mathrm{classified} \mathrm{by} \mathrm{Crawford} \mathrm{(1963)} \mathrm{and} \mathrm{Cowley}$ et al. (1969) using $U B V$ photometry as an Ap 0 star. Crawford et al. (1973) confirmed this spectral type with $u v b y \beta$ photometry. Molnar (1972) cassified HR 7224 as Bp 9.5 III Si star based on low-resolution spectroscopy. He notes, however, that the photometric spectral type according to the values of $B-V=-0.15, U-$ $B=-0.54$ given by Crawford and by Cowley et al. should be B5. Oja (1983) measured comparable values of $B-V=-0.18$, $U-B=-0.56$

\footnotetext{
* Based on observations obtained at the 2-m telescope at the Thüringer Landessternwarte Tautenburg and at the $1.8-\mathrm{m}$ telescope at the Bohyunsan Optical Astronomy Observatory.

$\star \star$ Individual radial velocities are only available in electronic form at the CDS via anonymous ftp to

cdsarc.u-strasbg.fr $(130.79 .128 .5)$ or via

http://cdsweb.u-strasbg.fr/cgi-bin/qcat?]/A+A/457/1033
}

HR 7224 was found to be a photometric variable by Winzer (1974) who deduced a period of 1.1663 d. Adelman (1997a) derived a period of $1.123095 \mathrm{~d}$ based on Strömgren uvby observations taken in 1993-95 with the Four College Automated Photometric Telescope (FCAPT). He found that the light curves in all four colors are in phase and show a double-wave with two maxima of different height. There was only one attempt to measure a magnetic field of HR 7224 by Bohlender et al. (1993) but without any findings.

Adelman (2004) reported extraordinary photometric changes of HR 7224. Comparing time series observations taken with FCAPT before 1996 with those taken in 2003 he found that the amplitude of variability increased from $0.04 \mathrm{mag}$ to $0.21 \mathrm{mag}$ and that the main period of variability appears to be of the order of $101 \mathrm{~d}$ rather than the known $1.123 \mathrm{~d}$. This astonishing statement as well as the fact that there has been no high-resolution spectroscopy made of the star so far encouraged us to start spectroscopic observations. The observed high amplitude of radial velocity (RV) variations make the star an ideal candidate for Doppler imaging and let us extent our observations towards time 
Table 1. Journal of TLS observations. In the first two columns we give, for each group of observations, mean JD and total number of observations. In the next columns JD, number of spectra, and exposure time $\Delta t$ are listed for each run separately.

\begin{tabular}{rr|rrr|rrr|rrr|rrr}
\hline \hline JD $_{\text {mean }}$ & $N_{\text {total }}$ & \multicolumn{1}{|c|}{ JD } & $N$ & $\Delta t$ & JD & $N$ & $\Delta t$ & JD & $N$ & $\Delta t$ & JD & $N$ & $\Delta t$ \\
\hline 3243 & 4 & 3240 & 1 & 1200 & 3241 & 1 & 1200 & 3243 & 1 & 1200 & 3246 & 1 & 1200 \\
\hline 3337 & 11 & 3331 & 1 & 1200 & 3332 & 1 & 1800 & 3333 & 1 & 1800 & 3334 & 1 & 1800 \\
& & 3335 & 2 & 1800 & 3336 & 1 & 349 & 3339 & 1 & 1800 & 3343 & 1 & 1800 \\
& & 3344 & 1 & 1800 & 3345 & 1 & 1800 & & & & & & \\
\hline 3451 & 144 & 3450 & 76 & 360 & 3451 & 68 & 360 & & & & & \\
\hline 3519 & 113 & 3518 & 49 & 360 & 3519 & 35 & 360 & 3520 & 29 & 360 & & \\
\hline 3548 & 73 & 3546 & 20 & 360 & 3548 & 21 & 360 & 3549 & 32 & 360 & & \\
\hline 3606 & 232 & 3601 & 58 & 360 & 3602 & 24 & 360 & 3604 & 24 & 360 & 3607 & 26 & 360 \\
& & 3608 & 7 & 360 & 3609 & 22 & 360 & 3610 & 5 & 360 & 3611 & 66 & 360 \\
\hline
\end{tabular}

series. In this first part we investigate the RV and line profile variations of the star.

\section{Observations and data reduction}

We obtained 577 high-resolution spectra of HR 7224 with the Coude-echelle-spectrograph at the 2-m telescope of the Thüringer Landessternwarte (TLS) Tautenburg. Table 1 lists the journal of observations in groups of nearly consecutive nights. Starting with single exposures in 2004, we extended the observations in 2005 where we observed time series over 14 nights. Echelle spectra have a spectral resolution of 63000 and cover the wavelength range from 4760 to $7400 \AA$. Spectra were reduced using standard MIDAS packages. Reduction included bias and straylight subtraction, filtering of cosmic rays, flat fielding, optimum extraction of spectra, wavelength calibration by a ThAr lamp, correction of instrumental shifts by using telluric $\mathrm{O}_{2}$ lines for comparison, and normalization to the local continuum. As well as the TLS observations we obtained three spectra of HR 7224 with the Bohyunsan Observatory Echelle Spectrograph attached to the 1.8-m telescope at the Bohyunsan Optical Astronomy Observatory. These spectra cover a larger spectral range from 3600 to $10500 \AA$ with a spectral resolution of 45000 .

\section{Radial velocity and line profile variations}

\subsection{RVs from cross-correlation and period search}

HR 7224 is a broad-lined star with $v \sin i$ of about $100 \mathrm{~km} \mathrm{~s}^{-1}$. Almost all lines in the spectrum are blended. $\mathrm{RVs}^{1}$ were measured from cross-correlation with a template over a wavelength range from $4960 \AA$ (end of $\mathrm{H} \beta$ red wing) to $5880 \AA$ (where stronger telluric lines occur). The template was iteratively built from the observed spectra by starting with one arbitrary spectrum of HR 7224, determining the RVs of individual spectra, shifting all spectra according to their obtained RVs and coadding the shifted spectra to build a new template. The finally obtained accuracy is limited by the accuracy of the cross-correlation technique which is of $62 \mathrm{~m} \mathrm{~s}^{-1}$ on average for this broad-lined star. It is distinctly larger than the systematic errors from instrumental shifts that can be corrected with a typical accuracy of $35 \mathrm{~m} \mathrm{~s}^{-1}$ by using telluric lines.

For the period search we used the program PERIOD0 $4^{2}$. By using multiple frequency fitting in combination with

\footnotetext{
${ }^{1}$ Measured RVs are available in electronic form at the CDS via anonymous ftp to cdsarc.u-strasbg. fr

2 Copyright (c) 2004 David Lenz, Inst. of Astronomy, Univ. of Vienna.
}

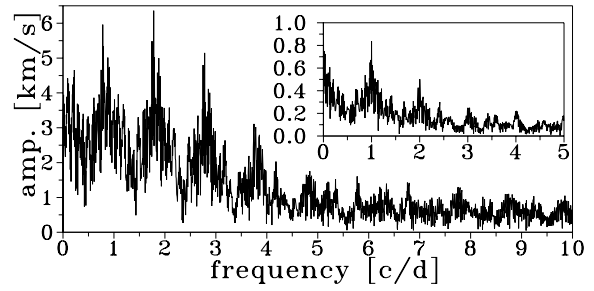

Fig. 1. Periodogram of observed RVs showing the highest peak at $1.78 \mathrm{c} \mathrm{d}^{-1}$ corresponding to the first harmonic of the deduced rotation period. The subframe shows the window function of data.

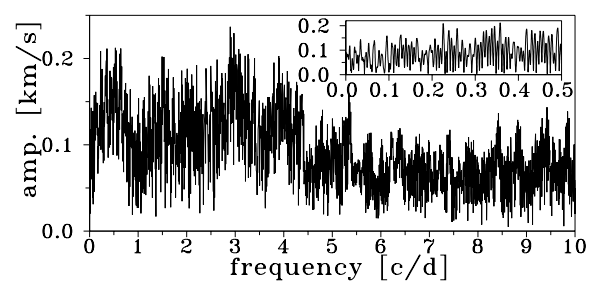

Fig. 2. Periodogram of the residuals after cleaning the data for the rotation period and its first three harmonics. The subframe shows the lowfrequency region.

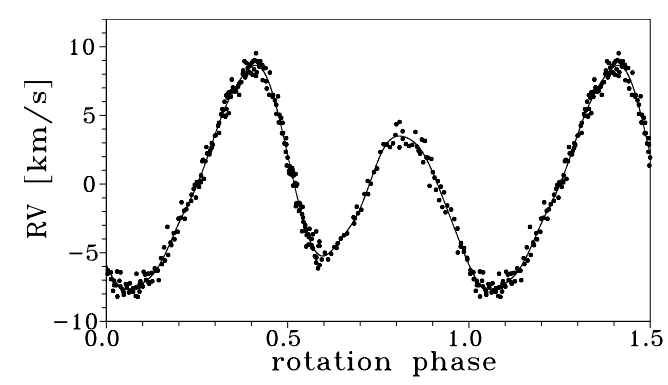

Fig. 3. Relative RVs of HR 7224 determined from cross-correlation and folded with the rotation period. The solid curve shows the variation calculated from the rotation period and its harmonics.

successive prewhitening of data we found significant peaks in the periodograms at a period of $1.123248 \mathrm{~d}$ and at positions corresponding to the first 3 harmonics of this period (Fig. 1). In the residuals of this solution (see Fig. 3) we found a further, spurious period of $0.312176 \mathrm{~d}$. Since this period is of the order of the first harmonics of the rotation period we do not believe in its physical significance. No further periodicities were found in the residuals, in particular not in the low frequency region around the $100 \mathrm{~d}$ period (Fig. 2) as observed by Adelman (2004). In the following we assume for HR 7224 a spectroscopic rotation period of $1.123248 \pm 0.000009 \mathrm{~d}$. The epoch of maximum RV is $2453520.51 \pm 0.05$. 


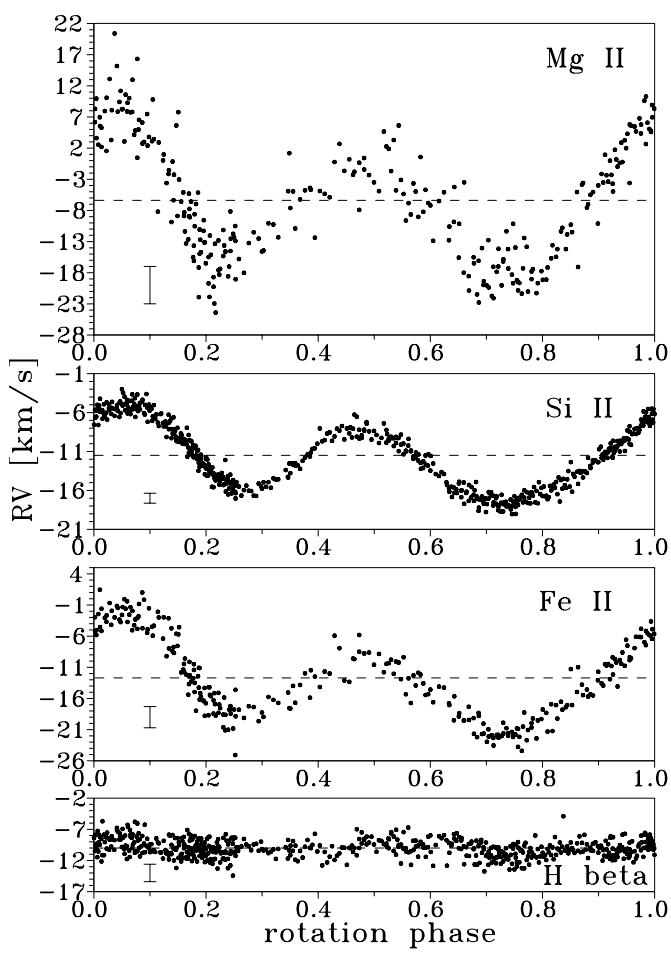

Fig. 4. RVs of HR 7224 determined from lines of single elements and folded with the rotation period. Systemic velocities are marked by dashed lines, mean errors of measurement by single error bars.

\subsection{RVs of individual elements}

In a next step we measured RVs of different elements by determining the centroids of individual lines using Gaussian fits. For metal lines we determined not only the centroid positions but also line depths and line widths as a function of rotation phase. For Si II we used the 5041, 5056, 5185, 6239, 6347, and $6371 \AA$ lines. The $6347 \AA$ line is slightly blended with Mg II, all other lines with Fe II. Further lines suitable for RV measurement were one Fe II line at $5316 \AA$, one Mg II line at $5264 \AA$, and one He I line at $5875 \AA$. These lines were selected because they were the only ones that fulfilled two selecion criteria: lines should have "single line" profiles (no blends with stronger neighbouring lines in the wings) and they should represent the corresponding element in the sense that the profile (blend) is dominated by the line(s) of this element.

$\mathrm{RV}$ s of Fe II lines were derived from a cross-correlation over the spectral range 5065-5182 $\AA$ that is free of contributions from other elements. Results are much less noisy than those obtained from the single $5316 \AA$ line. This line was used to determine the $\mathrm{Fe}$ II line depth variation, however. The selected He I line was too weak for measurement and the results showed only scatter. $\mathrm{RV}$ s were further determined from the $\mathrm{H} \beta$ profile using a fit of combined Cauchy and Gaussian profiles.

Results are shown in Figs. 4 to 6. Table 2 lists the mean systemic RVs derived from a fit of the RVs of each element by a combination of four sinusoids containing the orbital period and its first three harmonics. Listed amplitudes give the differences between observed maximum and minimum RVs. From Fig. 4 it is seen that the RVs of all metal lines vary in phase with each other. $\mathrm{H} \beta \mathrm{RVs}$ are constant. Figure 5 shows the relative variation of line depths (in terms of mean line depth) for elements Mg II, Si II, and Fe II. As in the case of RVs, the line depths of all elements vary in phase to each other. The amplitudes of the

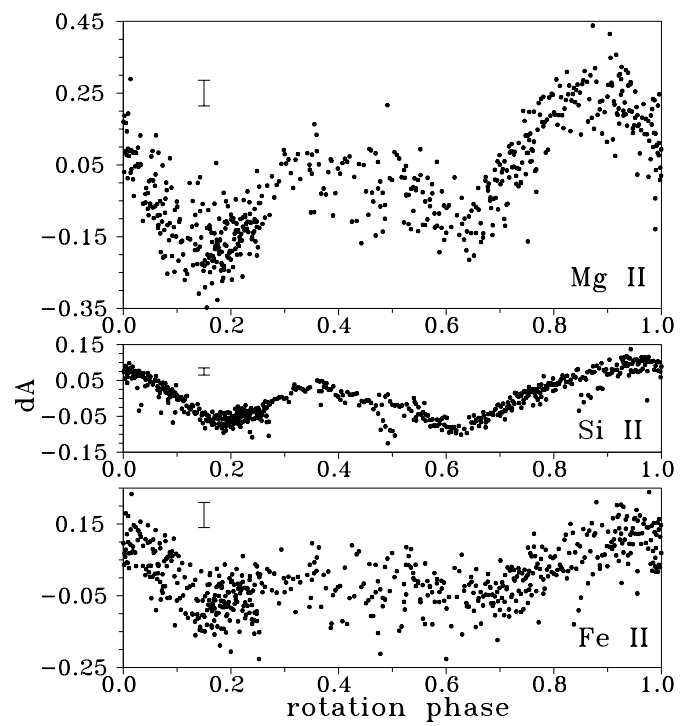

Fig. 5. Relative change of line depths folded with the rotation period.

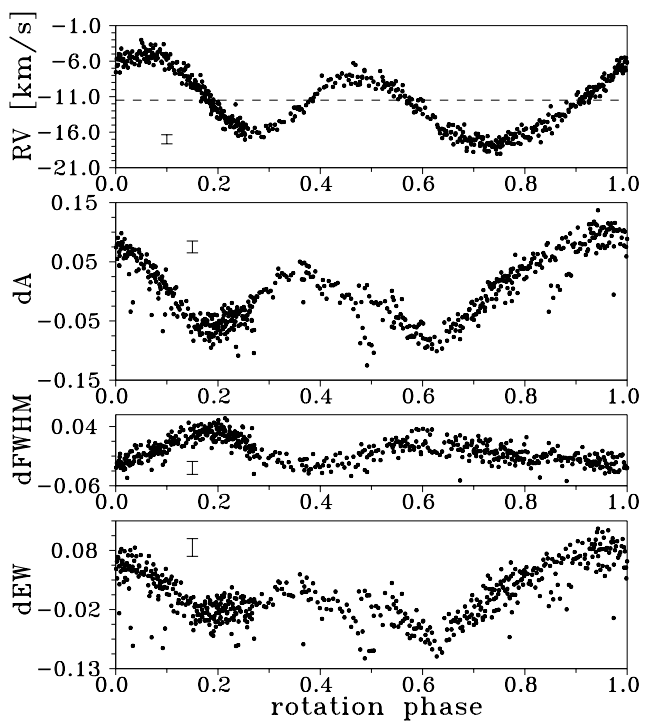

Fig. 6. From top to bottom: RVs, relative changes of depths, widths, and equivalent widths of silicon lines in HR 7224.

Mg II RV and line strength variations exceed that of silicon and iron by a factor of 2 .

Only for silicon was the accuracy of line width measurement sufficient to see a rotation dependent variation. Figure 6 compares the Si II variations of RV, line depth (in terms of mean line depth), and line width (in terms of mean line width). It further shows the eqivalent widths (EWs) computed from line depths and widths. Line width and line depth vary in antiphase. All minima and maxima of line width and line depth coincide with those phases of the RV variation where this variation has its mean values. The anti-correlation of line depths and widths results in a somewhat flatter variation of EWs compared to that of line depths.

\section{Possible surface configurations}

The observed period of $1.12324 \mathrm{~d}$ is in the range of typical rotation periods of stars of spectral type A to B as well as in the range of the pulsation periods of SPB stars (0.5 to $5 \mathrm{~d})$. Briquet et al. (2004) investigated four Bp variable stars with $\mathrm{He}$ and 
Table 2. Mean systemic radial velocities and amplitudes of RV variations for individual elements.

\begin{tabular}{lcccc}
\hline \hline & Mg II & Si II & Fe II & $\mathrm{H} \beta$ \\
\hline systemic velocity $\left[\mathrm{km} \mathrm{s}^{-1}\right]$ & -6.4 & -11.5 & -12.7 & -10.0 \\
amplitude $\left[\mathrm{km} \mathrm{s}^{-1}\right]$ & 28 & 13 & 19 & 0 \\
\hline
\end{tabular}

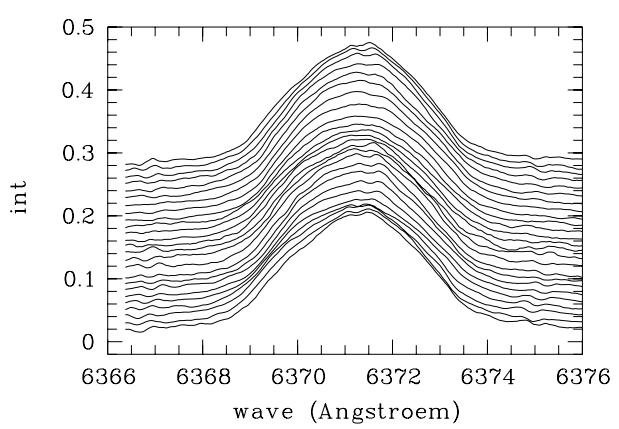

Fig. 7. Observed profiles (shown as line depths) of the Si II 6371 A line for 25 rotation phase bins.

Si surface inhomogeneities that show very similar behaviour to HR 7224 in many aspects. Following the arguments given in this paper we can exclude that the variations observed for HR 7224 are caused by pulsation: RV, line strength and line width variations show a double-wave with different heights of extrema and higher harmonics are present. Moreover, the observed difference in amplitudes of RV and EW variations of a factor of two between different elements can hardly be explained by pulsation. The strongest argument against pulsation is the observed constancy of $\mathrm{H} \beta \mathrm{RVs}$. For pulsations of early type stars, except for the roAp stars, we would expect the largest amplitude of RV variation from the Balmer lines. We therefore attribute the observed variations to surface inhomogeneities.

Figure 7 shows the observed line profile variation (LPV) of the Si II $6371 \AA$ line based on the mean averaged spectra that were obtained from 25 rotation phase bins. It is representative of the variation of all stronger Si II lines of HR 7224. Despite the large amplitude of RV variation of $\pm 6 \mathrm{~km} \mathrm{~s}^{-1}$ that indicates the presence of large spots of possibly high contrast, sharp bumps moving across the profiles are obviously absent. At first sight only a modulation of line depths can be seen. The question arises whether such LPV together with the observed variations in RV and EW are compatible with the assumption of a spotted surface. Here we try, in a first step, to find out if any spot configuration exists that can reproduce the observed LPV as well as the RV and EW variations. Such Doppler imaging of HR 7224 will be the content of a forthcoming paper.

The double-wave structure of RV and EW variations shown in Fig. 6 can be explained most simply by a surface structure that includes two larger spots. If spots are on opposite sides and are of the same size and same strength we would obtain a sinusoidal variation of both RV and EW. A closer look at Fig. 6 shows that the heights of the two maxima as well as the depths of the two minima of the RV variation are different. Also, the heights of the two maxima of the line depths (and EW) variation are different whereas the difference of the two minima is only marginal and all curves are slightly asymmetric (not really sinusoidal). In any case, the phase correlation between RV and EW variations would look like the observed one: if one of the spots is in the center of the star, the RV would at its mean value and $\mathrm{EW}$ is maximum. The amplitude of RV and EW variation can be

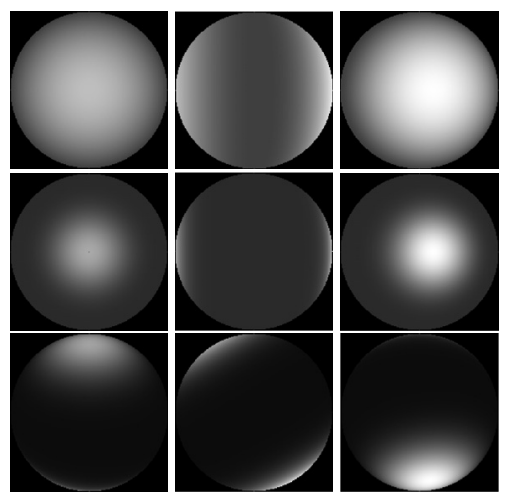

Fig. 8. Assumed spot configurations for rotation angles $\phi=0^{\circ}, 90^{\circ}$, and $180^{\circ}$. Rows from top to bottom show models 1,2 , and 4 .

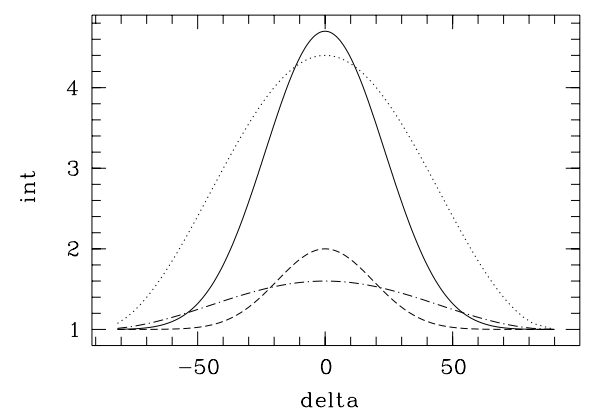

Fig. 9. Cross-sections of spot profiles. Different line types show models 1 (dash-dot), 2 (dashes), 3 (dotted), and 4 (continuous).

changed by changing the size or the strength of the spots as well as by different positions in latitude. A difference in the amplitudes of the two minima in EW occurs only if the two spots are not exactly on opposite sides in longitude. Different positions in longitude also change the symmetry of the observed RV and EW curves. In establishing a surface structure model we have also to take the shape of LPV into account. This has consequences for the size of the spots and the contrast between spots and the nonspotted surface.

We computed line profiles assuming, in a first approximation, that lines emerging from a spot have the same intrinsic line profile shape as that from the non-spotted surface. For each surface point on the visible disc we calculate the wavelength shift due to stellar rotation according to the observed $v \sin i$. The line depth of the intrinsic profile emerging from that surface point is scaled according to the assumed distribution of line strength (see Fig. 8). For the intensity distributions we assumed half-waves of sinusoids having an extension of $180^{\circ}$ in latitude and longitude of the star. To control the sharpness of spots these sinusoids were folded with 2D-Gaussians. Cross-sections of model spots are shown in Fig. 9. Finally, we integrated over all wavelengthshifted and depth-scaled profiles over the visible disc to obtain one rotation-broadened profile per rotation phase bin.

For comparison we computed four models of different sharpness of spots, two for the equatorial case and two for spots at a latitude of $60^{\circ}$. Model parameters are listed in Table 3. Amplitudes of spots are given in terms of the ratio of maximum to minimum line strength. In each case we adjusted the difference in longitude as well as the amplitudes of spots to fit the observed RV variation. Figures 10 and 11 compare the results for selected model configurations with the observed variations.

In general, models show the following: the basic shape and amplitude of observed RV variation can be reconstructed by all 
Table 3. Parameters of the four different surface configurations. We list latitude $\delta$ of spots, difference in longitude $\Delta \phi$ between the two spots, relative amplitudes $a$, and $F W H M$.

\begin{tabular}{crcccc}
\hline \hline model & $\delta\left[^{\circ}\right]$ & $\Delta \phi\left[^{\circ}\right]$ & $a_{1}$ & $a_{2}$ & $F W H M\left[{ }^{\circ}\right]$ \\
\hline 1 & 0 & 170 & 1.4 & 1.6 & 92 \\
2 & 0 & 170 & 1.6 & 2.0 & 44 \\
3 & 60 & 165 & 3.3 & 4.4 & 92 \\
4 & 60 & 165 & 3.3 & 4.7 & 54 \\
\hline
\end{tabular}
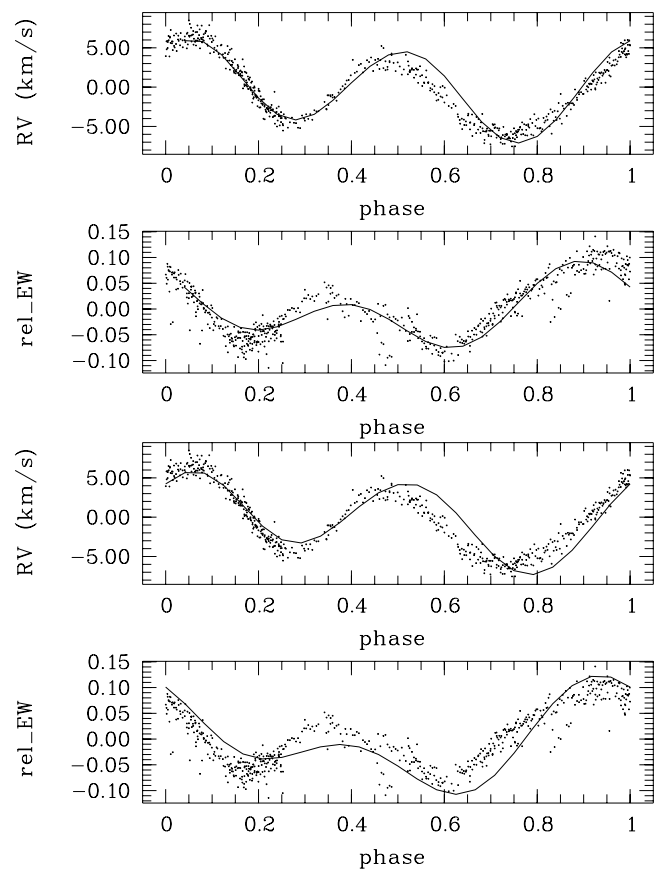

Fig. 10. Comparison of RV and EW variations computed from model 1 (upper two panels) and model 4 (lower panels) with measured values.

four models, that means independent of latitude and sharpness of spots. Spots at higher latitudes must have stronger contrast (Table 3). Equatorial spot configurations fit the observed EW variation slightly better (Fig. 10). To obtain a sufficiently smooth LPV the spots must be very extended. Even sinusoid-like spots with an extension over the full visible disk (model 1) show a more structured LPV than the observed one (Fig. 11). There is a discrepancy between the observed flat LPV and the high amplitudes in RV and line strength variations.

\section{Spectral type and abundance determination}

To estimate $T_{\text {eff }}, \log g, v \sin i$ and elemental abundances for HR 7224 we used the LLmodels code by Shuliak et al. (2004) for the computation of model atmospheres and the SynthV code by Tsymbal (1998) for the computation of synthetic line spectra. The model atmosphere code allows us to calculate 1D planeparallel, LTE atmospheric models for F to B stars near the main sequence. Line absorption can be calculated directly for each line from a preselected line list, making allowance for individual abundance patterns. SynthV is a non-magnetic spectrum synthesis code. Individual abundances are supported. The line list was taken from the VALD data base (Kupka et al. 2000) in its version of Nov. 2005.

For analysis we averaged the 577 obtained spectra of HR 7224 to build 25 rotation phase bins. Then we investigated the mean spectra $b_{\min }$ and $b_{\max }$ from the two bins with lowest
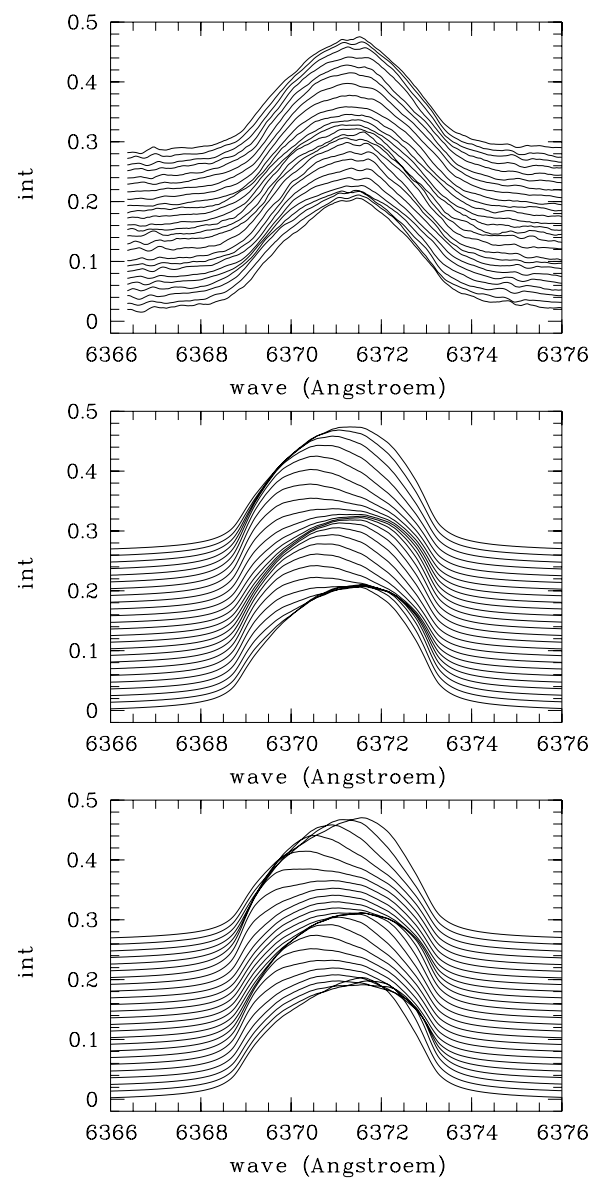

Fig. 11. Line depth profiles of the Si II $6371 \AA$ line for 25 rotation phase bins. From top to bottom: observed profiles, model 1, model 2.

and highest strength of silicon lines (see Fig. 6 at phases 0.6 and 0.9 ), respectively. Model values of $T_{\text {eff }}$ depend on assumed metallicity and in particular on the assumed helium and silicon abundances. On the other hand, to derive these abundances from observed spectra, we have to apply a model atmosphere with given $T_{\text {eff }}$. We tried to solve this problem by determining $T_{\text {eff }}$ and $\log g$ from the Balmer line profiles, calculating a corresponding model atmosphere and to fit the resulting synthetic spectrum to the metal lines of HR 7224 to determine $v \sin i$ and individual metal abundances. Then we repeated this procedure by calculating new model atmospheres based on the derived abundances to derive new values of $T_{\text {eff }}$ and $\log g$ from the Balmer line fits.

Blends due to the large $v \sin i$ of HR 7224 complicate the abundance analysis. To derive e.g. the silicon abundance from a single Si II line we had to fit the iron abundance for all Fe II lines in the neighbourhood of the Si II line. Synthetic spectra were computed for the main lines of Si II, He I, and Mg II. For Fe II and $\mathrm{S}$ II we computed mean abundances from the wavelength region 4960-5600 A. No contributions from other elements could be identified in the spectra.

After iterating the described procedure we end up with a $v \sin i$ of $100 \pm 2 \mathrm{~km} \mathrm{~s}^{-1}$ and the Balmer line fits as shown in Fig. 12. Synthetic spectra practically coincide with the observed ones. Model parameters $T_{\text {eff }}$ and $\log g$ were obtained from the rms of the sum of the residuals from both $\mathrm{H} \alpha$ and $\mathrm{H} \beta$ line profile fits. We obtain $\log g=4.18 \pm 0.05$ and different temperatures for the two rotation phases: $T_{\text {eff }}=15500 \mathrm{~K}$ for the phase of minimum line strength and $T_{\text {eff }}=14500 \mathrm{~K}$ for the maximum phase. The difference of about $1000 \mathrm{~K}$ is remarkable. To 

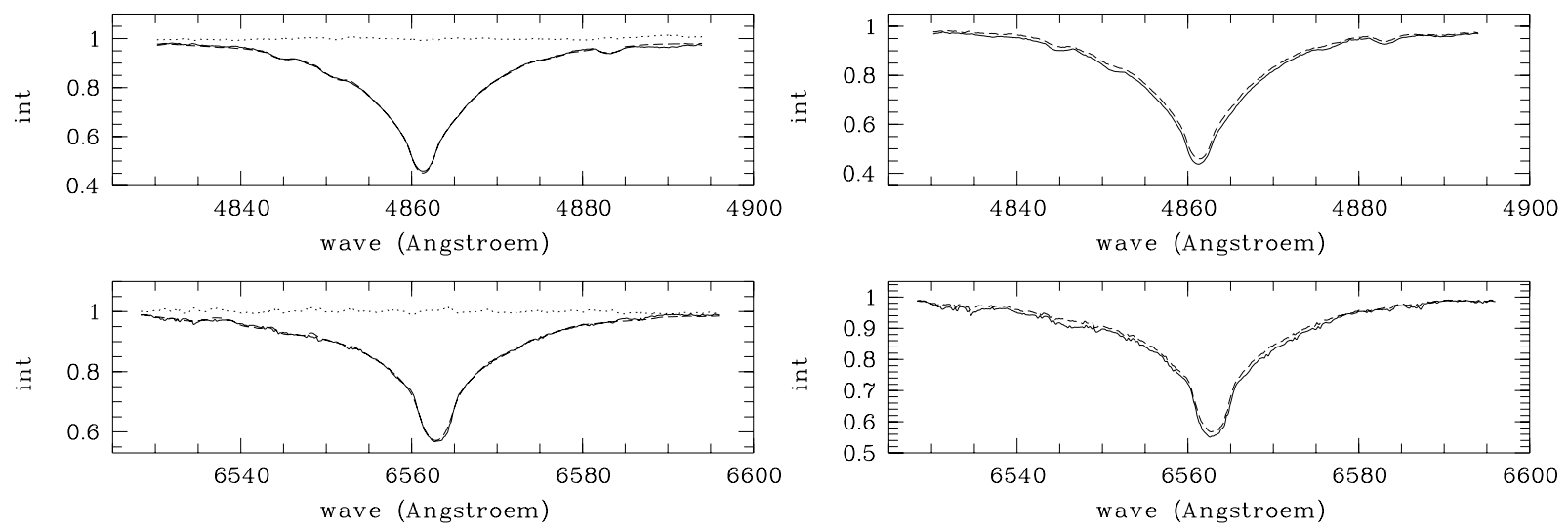

Fig. 12. Left panels: Balmer line profiles at phase of minimum line strength (solid curves) fitted by synthetic profiles (dashed curves) with $T_{\text {eff }}=15500 \mathrm{~K}, \log g=4.18$. Dotted curves show the residuals. Right panels: comparison of Balmer lines taken from phase of maximum (solid curves) and minimum (dashed curves) line strength.

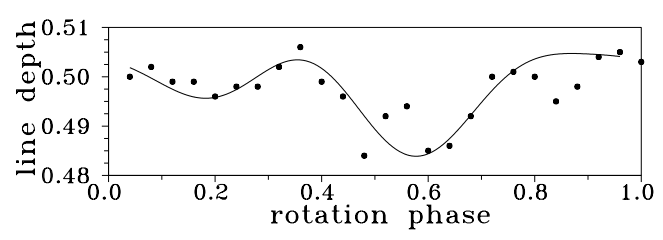

Fig. 13. Variation of central line depths of Balmer lines folded with the rotation period and fitted by sinusoids including the rotation period and its first two harmonics.

estimate the error of the temperature determination we plotted the observed line depths of Balmer lines (mean of $\mathrm{H}_{\alpha}$ and $\mathrm{H}_{\beta}$ ) versus rotation phase (Fig. 13). The residuals of a fit by sinusoids including rotation period and the first two harmonics have a rms of 0.004 in line depth. Assuming a linear relationship between line depth and $T_{\text {eff }}$ over the $1000 \mathrm{~K}$ range we can estimate an error of $T_{\text {eff }}$ measurement of $\pm 200 \mathrm{~K}$. Thus the effective temperatures for phases of minimum and maximum line strength differ significantly, we get $\Delta T_{\text {eff }}=(1000 \pm 400) \mathrm{K}$.

As an additional test we computed synthetic line profiles of the Si II $5041 \AA$ and the Mg II $5264 \AA$ lines from model atmospheres with $T_{\text {eff }}=14500$ and $15500 \mathrm{~K}$. Resulting profiles were folded with $v \sin i=100 \mathrm{~km} \mathrm{~s}^{-1}$ and rebinned to RV. Normalization was done in such a way that the central line depth of the averaged mean profiles from both temperatures is unity. Figure 14 shows the results. The ratio of central line depths or equivalent widths is 1.12 for the $\mathrm{Mg}$ II line and of 1.06 for the Si II line. This means that the relative change due to a temperature change by $1000 \mathrm{~K}$ for the $\mathrm{Mg}$ line is twice the relative change of the Si line. This agrees very well with the observed difference in amplitudes of line depth changes between $\mathrm{Mg}$ and Si lines (Fig. 5) which now can be explained by different temperature sensitivity.

If the star turns out to have a very strong magnetic field (Bohlender et al. (1993) made only one measurement) the different behaviour of the Si II and the Mg II lines could also be due to different magnetic enhancement of line strengths (e.g. Guenther et al. 1999). This can be ruled out, however, by investigating the transition terms of the two lines (Table 4). The magnetic sensitivities in terms of the effective Lande factors of both lines do not differ much.

Table 5 lists the results of the abundance analysis. Errors of measurement are 0.05 for single lines, 0.02 for the four strongest Si II lines and the 4960-5600 Å range, and 0.2 for the

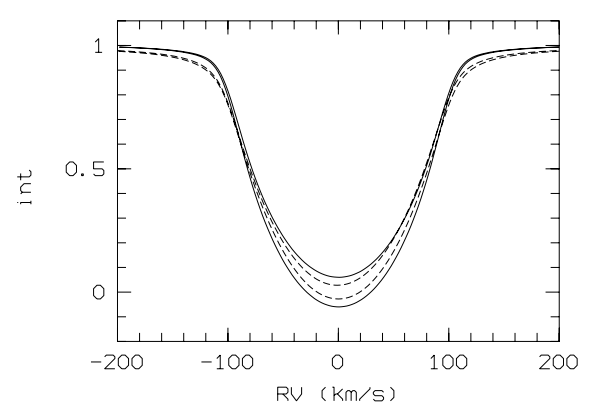

Fig. 14. Renormalized synthetic profiles of Mg II $5264 \AA$ (continuous) and Si II $5041 \AA$ (dashed) lines. In both cases the stronger profile corresponds to $T_{\text {eff }}=14500 \mathrm{~K}$, the weaker profile to $T_{\text {eff }}=15500 \mathrm{~K}$.

Table 4. Effective Lande factors $g_{\text {eff }}$ taken from Beckers (1969).

\begin{tabular}{ccc}
\hline \hline line & transition & $g_{\text {eff }}$ \\
\hline Si II 5041 $\AA$ & ${ }^{2} \mathrm{P}_{1 / 2}^{0}-{ }^{2} \mathrm{D}_{3 / 2}$ & 0.833 \\
Mg II 5264 & ${ }^{2} \mathrm{D}_{5 / 2},{ }^{2} \mathrm{~F}_{7 / 2}^{0}$ & 1.071 \\
& ${ }^{2} \mathrm{D}_{3 / 2}-{ }^{2} \mathrm{~F}_{5 / 2}^{0}$ & 0.900 \\
\hline
\end{tabular}

He I line. The obtained mean values are given in Table 6. In deriving these values, we gave values from Table 5 obtained for the four strongest silicon lines and all values obateined from the 4960-5600 A region twice the weight of other values.

It can be seen from Table 5 that there is a scatter in silicon abundances derived from different Si II lines. As already mentioned, we readjusted the iron abundance in each case by measuring the abundance of other elements to derive a best fit for iron lines in the nearest neighbourhood of measured Si II or other lines. During this adjustment iron abundances showed a scatter as well, comparable to that of silicon. No correlation of derived abundance values with the excitation potential of individual lines was observed. The differences of abundances derived for the two rotation phases do show such a correlation, however (Fig. 15).

Helium is extremely underabundand in HR 7224. Unfortunately the only measurable He I line in our spectra is very weak and heavily blended with a strong Si II line. That is why we have only a very rough estimate of line strength and abundance and observe no clear variation with the rotation cycle. An inspection of single spectra obtained with the Korean BOES spectrograph for the blue spectral region of HR 7224 confirms the extreme He deficiency. Figure 16 shows two He I lines fitted 
Table 5. Elemental abundances derived from individual lines. We list wavelength in $\AA$ ( $\lambda \lambda$ means the $4960-5600 \AA$ region), excitation potential in $\mathrm{EV}$, and derived abundances in phases of minimum and maximum line strength. He I values marked by * were derived from one BOES spectrum.

\begin{tabular}{|c|c|c|c|c|c|c|c|}
\hline$\lambda$ & $\Phi$ & $a_{\min }$ & $a_{\max }$ & $\lambda$ & $\Phi$ & $a_{\min }$ & $a_{\max }$ \\
\hline \multicolumn{4}{|c|}{$\overline{\text { Si II }}$} & \multicolumn{4}{|c|}{ Fe II } \\
\hline 5041 & 10.067 & -3.04 & -2.89 & 5316 & 3.153 & -3.68 & -3.69 \\
\hline 5056 & 10.074 & -3.16 & -2.98 & 5588 & 10.600 & -3.43 & -3.32 \\
\hline 5185 & 12.839 & -2.63 & -2.43 & 6247 & 10.909 & -3.57 & -3.42 \\
\hline 5466 & 12.526 & -3.12 & -2.94 & 6357 & 10.909 & -3.00 & -2.75 \\
\hline 5800 & 14.504 & -3.33 & -3.19 & $\lambda \lambda$ & - & -3.51 & -3.34 \\
\hline 5806 & 14.489 & -3.64 & -3.46 & \multicolumn{4}{|c|}{$\mathbf{S}_{\text {II }}$} \\
\hline 5868 & 14.528 & -2.99 & -2.82 & $\lambda \lambda$ & - & -5.31 & -5.20 \\
\hline 5915 & 14.528 & -3.42 & -3.27 & \multicolumn{4}{|c|}{ Mg II } \\
\hline 5957 & 10.067 & -3.08 & -2.86 & 5264 & 11.569 & -4.14 & -3.85 \\
\hline 5979 & 10.074 & -2.95 & -2.79 & $\lambda \lambda$ & - & -4.29 & -3.96 \\
\hline 6239 & 12.839 & -2.82 & -2.61 & \multicolumn{4}{|c|}{ He I } \\
\hline 6347 & 8.121 & -3.01 & -2.95 & 5876 & 20.964 & -3.47 & -3.04 \\
\hline 6371 & 8.121 & -3.05 & -2.97 & 4026 & 20.964 & \multicolumn{2}{|c|}{$-3.0^{*}$} \\
\hline$\lambda \lambda$ & - & -3.18 & -3.06 & 4471 & 20.964 & \multicolumn{2}{|c|}{$-3.5^{*}$} \\
\hline
\end{tabular}

Table 6. Final mean abundances. We give solar values, abundances at phases of minimum and maximum line strength, mean of min, max, difference of min-max, and difference of mean to solar abundance. For Si II and Fe II errors are given in brackets, in units of the last digit.

\begin{tabular}{lllllll}
\hline \hline el. & solar & \multicolumn{1}{c}{$\min$} & \multicolumn{1}{c}{$\max$} & mean & \multicolumn{1}{c}{$\Delta$} & $\Delta_{\odot}$ \\
\hline $\mathrm{He}_{\mathrm{I}}$ & -1.00 & -3.5 & -3.0 & -3.2 & 0.4 & -2.2 \\
$\mathrm{O}_{\mathrm{I}}$ & -3.28 & -3.2 & -3.1 & -3.1 & 0.1 & +0.2 \\
$\mathrm{Mg}_{\text {II }}$ & -4.57 & -4.2 & -3.9 & -4.0 & 0.3 & +0.5 \\
$\mathrm{Si}_{\text {II }}$ & -4.50 & $-3.1[2]$ & $-2.9[3]$ & $-3.0[2]$ & $0.15[5]$ & $+1.5[2]$ \\
$\mathrm{S}_{\text {II }}$ & -4.83 & -5.3 & -5.2 & -5.3 & 0.1 & -0.4 \\
$\mathrm{Fe}_{\text {II }}$ & -4.37 & $-3.3[3]$ & $-3.2[4]$ & $-3.3[4]$ & $0.02[1]$ & $+1.1[4]$ \\
\hline
\end{tabular}

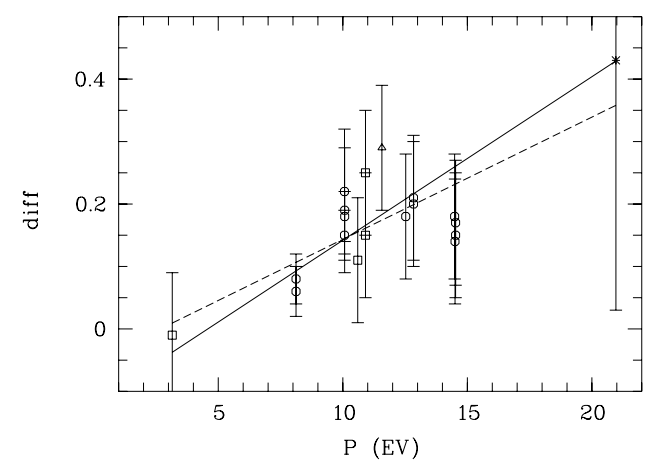

Fig. 15. Abundance difference $a_{\min }-a_{\max }$ versus excitation potential for Si II (circles), Fe II (squares), Mg II (triangle) and He I (asteriks) lines. Regression lines are plotted for weigthed (solid line) and unweighted (dashed line) data.

by model atmospheres assuming $\mathrm{He}$ abundances of -3.0 and -3.5 . One line profile resulting from solar abundance is shown for comparison. Besides the high deficiency Fig. 16 also shows that we obtain different $\mathrm{He}$ abundances from different lines (here with a difference of 0.5 ).

\section{Photometric colors}

We computed photometric colors and color indices from model atmospheres for the two rotation phases of minimum $\left(T_{\text {eff }}=15500\right)$ and maximum $\left(T_{\text {eff }}=14500\right)$ line strengths using

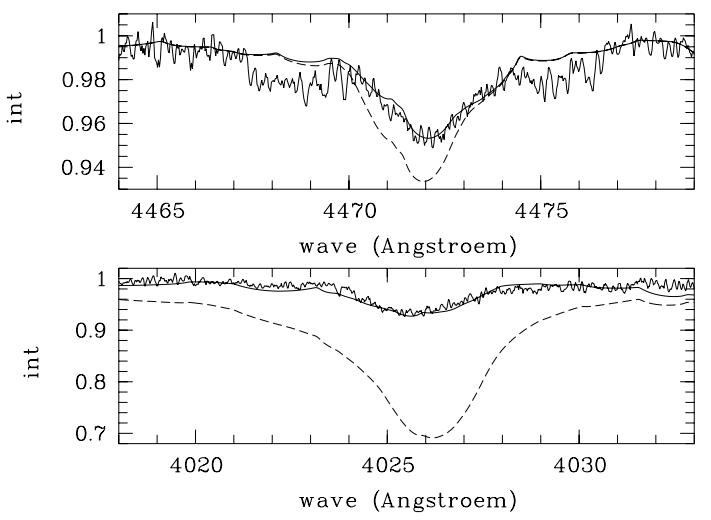

Fig. 16. He I lines from BOES spectra fitted by synthetic profiles assuming different He abundances. Top: $4472 \AA$ line, abundance of -3.5 (solid) and -3.0 (dashed). Bottom: $4026 \AA$ line, abundance of -3.0 (solid) and solar abundance (dashed).

Table 7. Color indices computed from the two model atmospheres of 14500 and $15500 \mathrm{~K}$ compared to data from the literature. Sources are Cowley et al. (1969) for $U B V$ values based on 1964 observations and Crawford et al. (1973) for Strömgren color indices obtained between 1965 and 1973.

\begin{tabular}{lccccccc}
\hline \hline source & period & $U-B$ & $B-V$ & $b-y$ & $m_{1}$ & $c_{1}$ & $\beta$ \\
\hline $15500 \mathrm{~K}$ & 2005 & -0.59 & -0.16 & -0.081 & 0.127 & 0.368 & 2.752 \\
$14500 \mathrm{~K}$ & 2005 & -0.53 & -0.15 & -0.077 & 0.137 & 0.426 & 2.764 \\
\multicolumn{1}{l}{ see caption } & -0.54 & -0.15 & -0.085 & 0.134 & 0.433 & 2.733 \\
\hline
\end{tabular}

Table 8. Color differences between the $14500 \mathrm{~K}$ and $15500 \mathrm{~K}$ atmospheres compared to the color amplitudes obtained by Adelman.

\begin{tabular}{lcllll}
\hline \hline source & period & $\Delta u$ & $\Delta v$ & $\Delta b$ & $\Delta y$ \\
\hline $14500-15500 \mathrm{~K}$ & 2005 & 0.20 & 0.13 & 0.12 & 0.11 \\
Adelman (1997a) & $1993-95$ & 0.055 & 0.039 & 0.035 & 0.027 \\
Adelman (2004) & 2003 & 0.21 & 0.20 & 0.20 & 0.19 \\
\hline
\end{tabular}

the computer code COLORS by Khan $\&$ the STARSP Group ${ }^{3}$ (see also Khan \& Shulyak 2006, Sect. 3.3). This code uses the flux distribution computed by LLmodels and calculates Johnson $U B V$ as well as Strömgren $u v b y \beta$ and Geneva colors.

Tables 7 and 8 list the results and compare them to previously obtained data. Our results of UBV color indices agree very well with those observed by Cowley et al. (1969) during an arbitrary rotation phase of HR 7224 (Table 7). There is also a good agreement of our calculated Strömgren $u v b y \beta$ color indices with those obtained by Crawford et al. (1973, Table 7). These observations were made between 1965 and 1973, the precise date was not given. Strömgren colors from the 1993-1995 period (Adelman 1997a) were taken from the published catalog (Adelman 1997b). 2003 data were kindly provided by Adelman (2004, 2006). Because all these values are based on differential photometry, they are not suitable to calculate color indices. They were only used to compare the amplitudes of variations. From Table 8 we see that the light curve amplitudes of the 2003 observations are comparable to our differences derived from the two rotation phase bins of minimum/maximum line strength, at least in order of magnitude. 1993-95 amplitudes are distinctly lower. We do not have data from other early observations but Adelman (1997a) stated that HR 7224 was detected to be

\footnotetext{
${ }^{3}$ http://www. starsp.org
} 
variable by Winzer (1974) who measured a maximum amplitude in Johnson $U$ of 0.05 mag.

\section{Conclusions}

Our spectroscopic campaign to examine HR 7224 was stimulated by the paper of Adelman (2004) where he supposed a drastic increase of rotation period from $1 \mathrm{~d}$ to about $100 \mathrm{~d}$ and of the photometric amplitude by a factor of six. Our results based on synthetic uvby colors calculated from spectra observed in 2004 and 2005 confirm these higher amplitudes. The amplitude in $u$ of 0.21 mag agrees very well with the amplitude observed by Adelman in 2003. Amplitudes calculated for $v b y$ are almost twice of the 2003 values (Table 8).

We have no physical explanation for the $100 \mathrm{~d}$ period observed in the FCAPT light curves from 2003. We performed an independent period search in these data. The most prominent peak in the periodogram is indeed at about $100 \mathrm{~d}$ and we did not find a period very near to the known rotation period of $1.123 \mathrm{~d}$. Additionally, however, peaks occur very near to $1 \mathrm{~d}$ that cannot be distinguished from the one day alias period. We conclude that it is more likely that in 2003 the star showed a variation with a period significantly different from the known rotation period and that the time sampling of the FCAPT data is not suitable for an unambiguous determination of such a period near to one day. Our data, on the other hand, are not well enough sampled to search for long periods in the range of $100 \mathrm{~d}$. We can only show that the RV variations observed in our data can be explained by the same rotation period that is well known from the star's photometry before 2003 and that the assumption that the star changed its rotation period by orders of magnitude cannot be true.

From the RV variation we measured a period of $1.12325 \pm$ $0.00001 \mathrm{~d}$ and observed the same double-wave curve as already known from photometry. All deviations in the shape of this curve from a sinusoidal one can be described by higher harmonics of the derived rotation period. We found no hints of other contributions of lower or higher frequency in the residuals of this solution. This means in particular that the surface structure of the star was stable within the period of observations of about one year. We conclude that there must have been a singular transient phase between 1995 and 2003 where the increase in photometric amplitude occured.

HR 7224 was classified as a A0p star. From our abundance analysis it follows that the mean abundances of $\mathrm{Si}$ and $\mathrm{Fe}$ are enhanced whereas He is strongly depleted (Table 6). Neglecting its chemical peculiarity, measured $U B V$ colors of HR 7224 refer to a mid B-type star as already stated by Molnar (1972). Our model atmosphere fit gives a mean $T_{\text {eff }}$ of $15000 \mathrm{~K}$ corresponding to spectral type B5. The derived $\log g=4.18$ contradicts the assumption by Molnar that the star is an evolved LC III star.

The observed phase relation between RV and line strength variations of the metal lines is in good agreement with those expected from a rotating spotted stellar surface. Models of surface structure in terms of Si II line strength show that there exist very simple spot configurations that can reproduce the observations. From the results obtained in Sect. 4 we can conclude that the stellar surface must be dominated by two spots of very large size that are placed at nearly opposite sides of the star and that must have very smooth transitions. The observed very flat structure of the LPV could not be reproduced by our spot models, however.

Our simple model describes the surface configuration of HR 7224 in terms of line strength. Line strength inhomogeneities can be caused by different local abundance concentrations but also by different temperatures in different regions. Three results of our investigation point towards an interpretation in terms if different temperature: First, HR 7224 shows line strength and RV variations of all metal lines as well as line strength variations of the Balmer lines. Second, line strength variations can be explained by atmospheric models of different effective temperature; the remaining abundance differences are only small. Third, the observed large difference in amplitudes of the RV and line strength variations between $\mathrm{Mg}$ II and Si II lines correlate with their different sensitivity to temperature changes.

The usual interpretation of the Balmer line variations of CP stars is that there is a variation of UV opacity due to the line blanketing effect caused by the enhancement of rare-earth elements. By backwarming, an opposite variation in the visible region is induced whereas the total flux remains constant and $T_{\text {eff }}$ stays stable (see e.g. Molnar 1973; Jamar 1979). On the other hand, surface temperature inhomogeneities had been reported by Kroll (1989) for the fast-rotating Ap star CU Vir and by BöhmVitense \& Van Dyk (1987) for $\alpha^{2}$ CVn. The authors find that $\alpha^{2}$ $\mathrm{CVn}$ is cooler by $1000 \mathrm{~K}$ at the magnetic poles than at the equator and explain the observed photometric changes by a nonspherically symmetric oblique rotator model. This explanation nicely compares with our observations of HR 7224 if we assume that the star has extended cool polar caps that are slightly inclined to the rotation axis (see our model 4 in Sect. 4). The question of why the star should be cooler at the magnetic poles is still unsolved, however. Since in the atmospheres of A- and B-type stars no convective energy transport takes place, the magnetic field cannot suppresss any convective energy flux and the analogy of solar spots does not work. We thus cannot speculate about the physical origin of temperature inhomogeneities on HR 7224 as we have no knowledge about its magnetic field.

Although our iterative $T_{\text {eff }}$ /abundance determination shows a difference in model $T_{\text {eff }}$ of about $1000 \mathrm{~K}$ and only marginally different $\mathrm{Si}$ and $\mathrm{Fe}$ abundances for rotation phases of minimum/maximum line strength, we cannot conclude that we have different integral fluxes from the star in different rotation phases. The atmosphere of HR 7224 may be better described by a unique temperature and stronger abundance inhomogeneities because of the many limitations of our method that may also give rise to the observed correlation between differences in abundances and excitation potentials of different lines (Fig. 15). The use of a homogeneous atmosphere model as a first approach, the unknown surface configuration of helium, the effects of possible stratification of He and Si abundances (see e.g. Leone \& Lanzafane 1997), and the possibly imperfect handling of Stark damping of the Si II lines contribute to the uncertainty. The effect of Stark damping on equivalent widths of Si II lines due to modified Stark widths was described by Lanz et al. (1988). There are no such investigations for Stark shifts, however, which may give rise to additional broadening of spectral lines observed in the integrated light from an inhomogeneous stellar surface.

In a forthcoming paper we will investigate the atmosphere of HR 7224 in more detail using two-component atmosphere models that include stratification of elemental abundance and microturbulence. Moreover, we plan to analyze spectra of sharp lined silicon stars to derive empirical values for the Stark damping of Si II lines and to obtain a more detailed picture of the stellar surface structure by Doppler imaging.

Acknowledgements. Authors wish to thank the referee, Dr. David Bohlender, for his very useful comments and Prof. Artie Hatzes for his careful proofreading of the manuscript. VT acknowledges support by the Austrian Fonds zur Foerderung der wissenschaftlichen Forschung (P17890) and by the BM:BWK 
(project COROT). DEM acknowledges his work as part of the research activity of the Astrophysical Research Center for the Structure and Evolution of the Cosmos (ARCSEC), which is supported by the Korean Science and Engineering Foundation.

\section{References}

Adelman, S. J. 1997a, A\&AS, 122, 249

Adelman, S. J. 1997b, VizieR On-line Data Catalog: J/A+AS/122/249

Adelman, S. J. 2004, MNRAS, 351, 823

Adelman, S. J. 2006, private communication

Beckers, J. M. 1969, Sacramento Peak Obs. Contr. 141 - Phys. Sci. Res. Papers 371, Sacramento: Peak Observatory and Air Force Cambridge Research Laboratories, 1969

Bohlender, D. A., Landstreet, J. D., \& Thompson, I. B. 1993, A\&A, 269, 355

Böhm-Vittense, E., \& Van Dyk, S. D. 1987, AJ, 93, 1527

Briquet, M., Aerts, C., Lüftinger, T., et al. 2004, A\&A, 413, 273

Cowley, A., Cowley, Ch., Jaschek, M., et al. 1969, AJ, 74, 375
Crawford, D. L. 1963, ApJ, 137, 530

Crawford, D. L., Barnes, J. V., \& Golson, J. C. 1963, ApJ, 137, 530

Guenther, E. W., Lehmann, H., Emerson, J. P., et al. 1999, A\&A, 341, 768

Jamar, C. 1979, A\&A, 56, 413

Kim, K. M., Jang, B. H., Han, I., et al. 2002, J. Korean Astron. Soc., 35, 221

Khan, S. A., \& Shuliak, D. V. 2006, A\&A, 448, 1153

Kroll, R. 1989, Rev. Mod. Astron., 2, 194

Kupka, F., Ryabchikova, T. A., Piskunov, N. E., et al. 2000, Baltic Astron., 9, 590

Lanz, T., Dimitrijevic, M. S., \& Artru, M.-C. 1988, A\&A, 192, 249

Leone, F., \& Lanzafane, A. C. 1997, A\&A, 320, 893

Molnar, M. R. 1972, ApJ, 175, 453

Molnar, M. R. 1973, ApJ, 179, 527

Oja, T. 1983, A\&AS, 52, 131

Shulyak, D., Tsymbal, V., Ryabchikova, T., et al. 2004, A\&A, 428, 993

Tsymbal, V. 1996, ASP Conf. Ser., 108, ed. S. J. Adelman, F. Kupka, \& W. W. Weiss, 198

Winzer, J. E. 1974, Ph.D. Thesis, Univ. Toronto 